\title{
Steroid Treatment for Recurrent Epididymitis Secondary to Idiopathic Urethritis and Urethrovasal Reflux
}

\author{
G. K. Ninan ${ }^{1}$ Preethi Bhishma $^{1}$ Ramnik Patel ${ }^{1}$ \\ ${ }^{1}$ Department of Paediatric Urology, Children's Hospital, University \\ Hospitals of Leicester NHS Trust, Leicester Royal Infirmary, Infirmary \\ Square, Leicester, United Kingdom \\ Eur J Pediatr Surg Rep 2013;1:56-59.
}

\begin{abstract}
Address for correspondence G. K. Ninan, FRCSG, FRCSI, FRCS Ed, FRCS (Paed Surg), Department of Paediatric Urology, Children's Hospital, University Hospitals of Leicester NHS Trust, Leicester Royal Infirmary, Infirmary Road, Leicester LE1 5WW, United Kingdom (e-mail: georgeninen@gmail.com).
\end{abstract}

\author{
Abstract \\ Keywords \\ - idiopathic urethritis \\ - urethrovasal reflux \\ - urethro-ejaculatory \\ reflux \\ - acute scrotum \\ - recurrent \\ epididymitis \\ - topical steroid \\ triamcinolone
}

We describe a case of recurrent left-sided epididymitis secondary to severe idiopathic posterior urethritis extending to left seminal vesicle and vas deference with associated urethrovasal reflux (UVR). Cystourethroscopy and micturating cystourethrogram were essential for the diagnosis. Following cystourethroscopy, intravesical, and urethral instillation of topical steroid triamcinolone, patient had a full recovery. Idiopathic urethritis in association with veru montentitis, utriculitis leading to left-sided UVR, inflammation of the seminal vesicle, and vas deference causing secondary epididymitis is rare. We report the first such rare case presenting as recurrent acute scrotum and response to innovative treatment we used.

\section{Introduction}

Nonspecific urethritis in children is rare and mild disease. ${ }^{1,2}$ Adolescent urethritis in most cases is self-limiting. ${ }^{3}$ To our knowledge, this is the first case in which an ascending posterior urethritis extending laterally into seminal vesicles and vas deference and causing inflammation of epididymis simulating acute scrotum in whom a battery of various investigations including micturating cystourethrogram (MCUG) and cystourethroscopy were a useful adjunct in establishing the diagnosis and an innovative management included intravesical instillation of triamcinolone was effective as the definitive treatment.

\section{Case Report}

A 12-year-old boy was first presented at a tertiary pediatric surgical hospital with a 20-hour history of left scrotal pain, dysuria, hematuria, and tenderness while he was on holidays. Urine dipstick and culture were negative. He underwent left

scrotal exploration that revealed no obvious abnormality. Left hydatid of Morgagni was excised and left orchidopexy was performed. He was given trimethoprim for 5 days, but he continued to have dysuria and left scrotal pain postoperatively.

He was then presented to urology and pediatric departments of his local general hospital with continued dysuria pain in the left scrotum with radiation to left loin and tender left epididymis 2 months later. Pain score could be 10/10 despite regular triple analgesia and antispasmodic medications. He had at times difficulty in walking with wide-based gait due to dysuria associated with left lower abdominal, pelvic-perineal, and scrotal pain. His urine dip was negative, had normal renal functions, full blood count, and biochemical bone profile. His C-reactive protein was less than 5 and erythrocyte sedimentation rate was $2 \mathrm{~mm} /$ hour. Abdominal X-ray revealed no evidence of renal calculi. He felt to be constipated and was treated with a disimpaction dose of Movicol Paediatric Plain (Norgine). Ultrasound scan showed both testes normal in size, echo texture, and vascularity. Left

(c) 2013 Georg Thieme Verlag KG Stuttgart · New York
DOI http://dx.doi.org/ 10.1055/s-0033-1343076. ISSN 2194-7619.

October 25, 2012 
epididymis was swollen with increased vascular flow in comparison with the right. Kidneys, ureters, and bladder were normal. The appearances were consistent with left epididymitis. He was treated with co-amoxiclav for 10 days. He was discharged home after 7 days but continued to have dysuria and severe left scrotal pain.

He was readmitted at his local hospital after 2 weeks with the same symptoms of dysuria and nonremitting pain in the left scrotum and left side of lower abdomen. Urinalysis revealed 115 white blood cells (WBCs), no red cells, and no subsequent growth on culture. The ultrasound scan of abdomen, renal system, and testes were normal. Both epididymides were enlarged, with the right more noticeable than the left with reduced echo texture and slightly increased Doppler flow suggestive of bilateral epididymitis. He was treated with 14 days of cefalexin with triple analgesia with paracetamol, ibuprofen, and codeine. Repeat urinalysis 2 days later showed WBC of 14 only.

He was again presented 3 weeks later to his local hospital with no improvement of symptoms. Repeat ultrasound of the abdomen, renal system, and testes was normal. There was bilateral epididymal thickening more marked on the left side with a 4-mm cyst noted in the right epididymal head and upper body of the left epididymis. Minor generalized subcutaneous tissue thickening noted in the left scrotum. He was, therefore, referred to us for further management for dysuria associated with chronic and recurrent epididymitis with disabling pain.

He was seen in clinic and an emergency admission was requested because of the severity of his symptoms that started approximately 3 months prior to this consultation. Urine tests revealed no infection, and several courses of antibiotics have not helped him resolve his pain.

The clinical examination; laboratory tests; radiological investigations; and abdominal, renal, and testicular ultrasounds were normal. Both epididymides were enlarged with left more prominent than right on scrotal ultrasound scan. A magnetic resonance urogram was performed, which showed bilateral simplex kidneys and no abnormality of renal or collecting system. Uncommon diagnosis of idiopathic urethritis and urethrovasal reflux was considered based on our previous experience.

At cystourethroscopy, he was found to have severe posterior urethritis with active inflammation of verumontanum and both ejaculatory ducts were swollen and red. There was a large inflamed utricle with inflamed and swollen verumontanum with debris in the posterior urethra and bladder. The left ejaculatory duct was open and freely refluxing into the seminal vesicle and vas deference (-Fig. 1). Triamcinolone $40 \mathrm{mg}$ diluted in $10 \mathrm{~mL}$ of water for injection was instilled topically into the urethra and bladder. He underwent MCUG that showed free reflux of the contrast into the left seminal vesicle and vas deference in the micturating film (-Fig. 2).

He was seen in follow-up clinic in 2 weeks and was very much better and walking normally. The scrotal pain has gone down to $1 / 10$ and $4 / 10$ on deep palpation of the left epididymis. At 6-week follow-up, he was well, asymptomatic, was not on any medication, and was back to school after a period of 3 months from the onset of illness. A follow-up cystoscopy and cystogram was considered but as the patient was completely
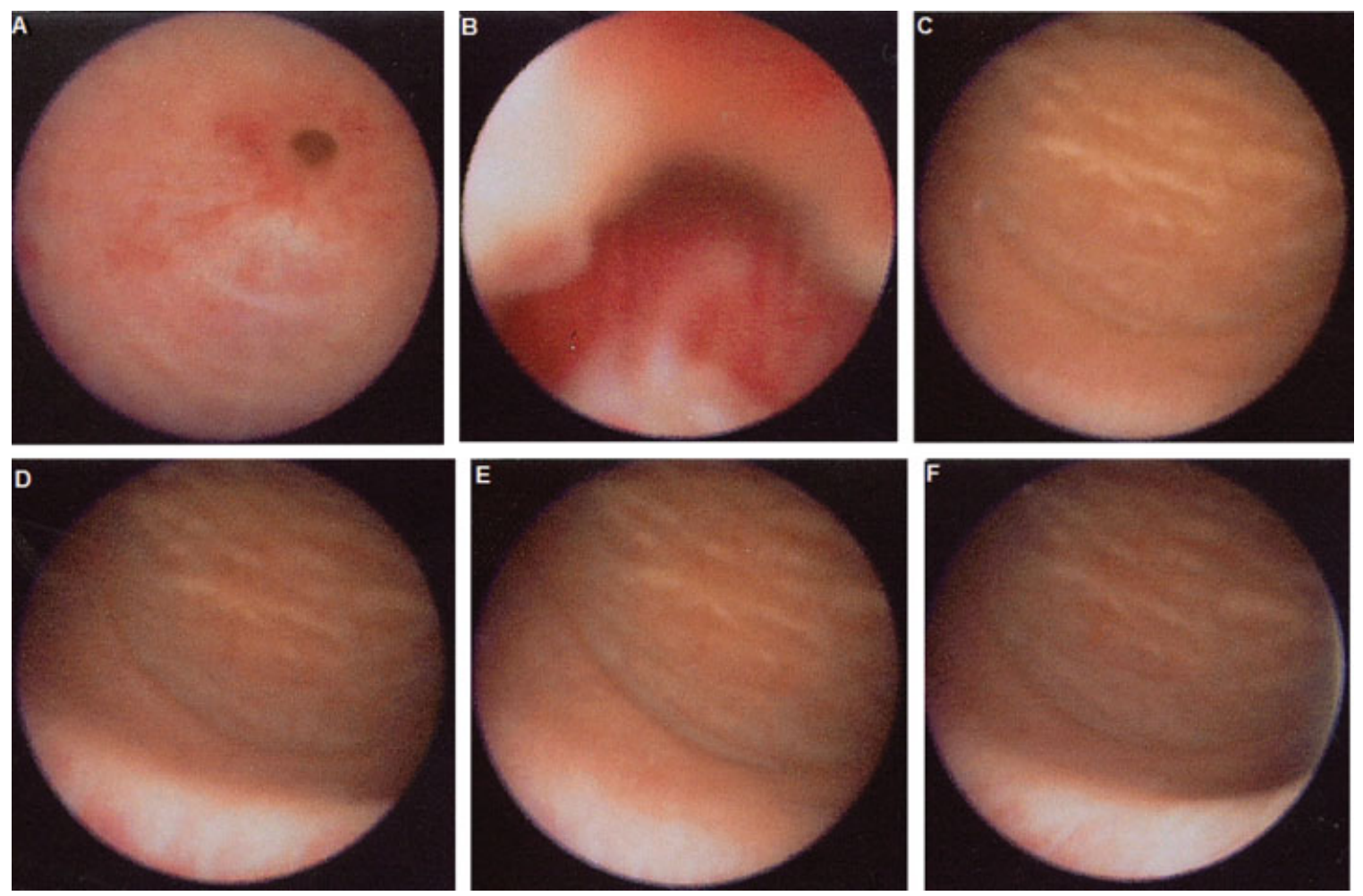

Fig. 1 Cystourethroscopic picture showing severe inflammation in and around verumontanum. (A) bulbous urethra, (B) posterior urethra, (C) bladder neck, (D) trigone, (E) ureteric orifices, (F) bladder. 


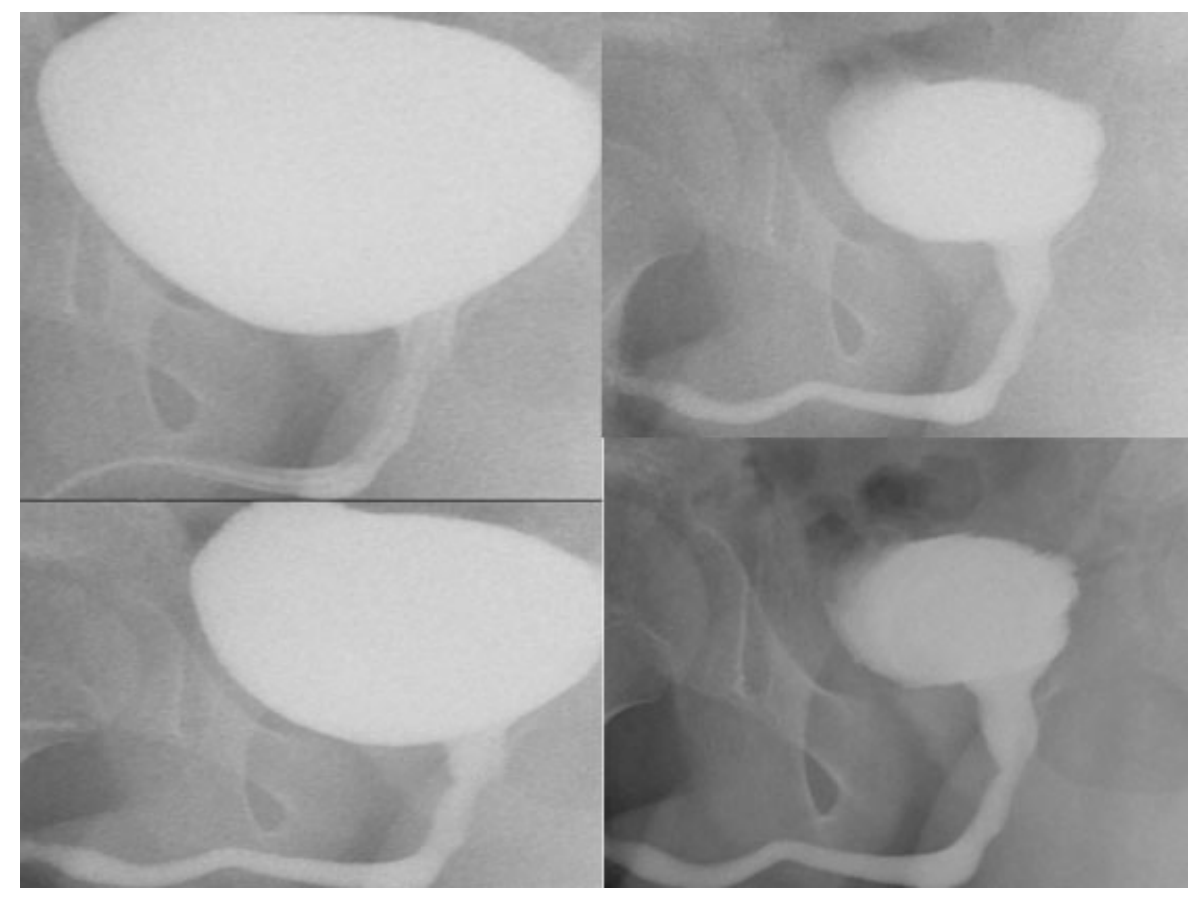

Fig. 2 Micturating cystourethrogram-note free reflux into the left vas deference from posterior urethra.

asymptomatic and well, the patient and parents were not happy to undertake it unless he gets recurrence in the long run. At 2-year follow-up, he is asymptomatic, both epididymides were normal and nontender, and the ultrasound scan showed complete reversal of thickening to normal on both epididymides. Patient, parents, referring pediatricians, and adult urologists were very pleased with the outcome.

\section{Discussion}

Idiopathic urethritis in male children is diagnosed by excluding bacterial infection and urethroscopy is helpful, but there is no specific treatment available. ${ }^{2}$ Bala Eradi and George Ninan (2009) for the first time reported use of novel therapeutic intervention of intravesical topical instillation of steroids as an effective treatment with complete reversal of symptoms and the local inflammation. ${ }^{4}$ Since then we had a large series of idiopathic urethritis patients treated with this novel therapeutic option with gratifying results. Most patients need one or two instillations and only one case with extension of inflammation into the bladder and ureters needed six instillations. The series of these patients with long-term follow-up is being reported separately.

Ascending spread and flare is well known in balanitis xerotica obliterans ${ }^{5}$; we could not find any case of nonspecific urethritis having ascending spread to the genital tracts with its urethrovasal reflux and spread of inflammation in association with the literature. The suggestion that the epididymal inflammation was secondary to posterior urethritis is interesting and suggests reflux or efflux of chemical, inflammatory, or infectious agent.

Reflux into the seminal vesicles, vas deference, and epididymis has been reported as postoperative complication following transurethral resection of the prostate in adults. ${ }^{6}$
Epididymitis is very rare in children, and occasional cases of vassal reflux as a cause of acute scrotum in children has been mentioned in English and non-English literature. ${ }^{7-9}$

Structural urologic abnormalities are common in children and in men older than 40 years with acute epididymitis. Adults usually have bladder outlet obstruction or urethral stricture. Children may have urethral abnormalities, such as a prostatic utricle, urethral duplication, posterior urethral valves, or urethrorectal fistula, or other anomalies, such as an ectopic ureter, ectopic vas deferens, detrusor sphincter dyssynergia, or vesicoureteral reflux. Siegel et al found that $47 \%$ of prepubertal boys with epididymitis had associated urogenital abnormalities, including ectopic vas deferens or ureters, and urethral abnormalities. ${ }^{10}$

Reflux of urine into the ejaculatory ducts of children may result in recurrent orchitis and sterility. Urethro-ejaculatory duct reflux (UER) is an uncommonly reported condition in children. The diagnosis of this condition can be made using a MCUG to demonstrate the reflux of contrast into any of the ejaculatory ducts. ${ }^{11}$

Symptoms usually are mild and periodic lasting between 1 and 4 weeks at a time, but in our patient, symptoms continued unabated for several months and needed several admissions and investigations, and fortunately got cured following intravesical instillation treatment of single topical steroid. Our patient needed an intervention with our novel therapeutic option to help him get out of the persistent symptoms disabling him from his school and requiring significant triple analgesia and number of antibiotics without any relief. We wonder how a single dose of topical steroid-even one with the pharmacokinetic profile of triamcinolone-would be effective in the long run while reflux persists. Earlier in the series, we have done follow-up cystoscopy and cystogram in a 
patient with posterior urethritis who had inflammation extending into upper urinary tract with complete reversal of the inflammation and vesicoureteral reflux.

Topical steroids are helpful in early inflammations; advanced inflammation may need oral and at times intralesional or parenteral steroids to control the severe cases. ${ }^{12,13}$ UER is more common than originally thought. All boys who present with urogenital symptoms should have an MCUG as part of the investigative workup and be scrutinized for UER. Management should aim at correcting any underlying anomaly and providing prolonged antibiotics, but vasectomy and the injection of a bulking agent should be considered. The indications for these newer forms of treatments are not clearly defined. ${ }^{11}$

In conclusion, our patient had a complicated history with nonspecific urethritis resulting in genital tract inflammation predominantly on the left side resulting in recurrent acute scrotum that was investigated with a variety of measures and finally diagnosed at cystourethroscopy and MCUG; it was treated with intravesical instillation of single steroid that resulted in complete resolution.

\section{Acknowledgments}

We are grateful to the pediatric and urology teams at the referring hospital to select us in view of our special interests and previous experience with complex and difficult cases.

\section{Conflict of Interest}

None

\section{References}

1 Harrison SC, Whitaker RH. Idiopathic urethritis in male children. Br J Urol 1987;59(3):258-260

2 Williams DI, Mikhael BR. Urethritis in male children. Proc R Soc Med 1971;64(2):133-134

3 Dewan PA, Wilson TM. Idiopathic urethritis in the adolescent male. Eur Urol 1996;30(4):494-497

4 Eradi B, Ninan GK. Intravesical steroid instillation-a novel therapeutic intervention for idiopathic urethritis of childhood. Eur J Pediatr Surg 2009;19(2):105-107

5 Jayakumar S, Antao B, Bevington O, Furness P, Ninan GK. Balanitis xerotica obliterans in children and its incidence under the age of 5 years. J Pediatr Urol 2012;8(3):272-275

6 Hübler J, Fariborz B, Fábos Z. An unusual complication of transurethral resection: reflux into the vas deferens, seminal vesicles and epididymis. J Urol 1999;162(5):1696

7 Kiviat MD, Shurtleff D, Ansell JS. Urinary reflux via the vas deferens: unusual cause of epididymitis in infancy. J Pediatr 1972;80(3): 476-479

8 Lima M, Ruggeri G, Grandi M, Dòmini M, Sciutti R, Tani G. [Urethrovesical-vas deferens reflux as a cause of acute scrotum]. Pediatr Med Chir 1992;14(3):323-326

9 Henriet R. [Scrotal urinary fistula due to urethro-vas deferens reflux]. J Urol Nephrol (Paris) 1976;82(6):523-524

10 Siegel A, Snyder H, Duckett JW. Epididymitis in infants and boys: underlying urogenital anomalies and efficacy of imaging modalities. J Urol 1987;138(4, Pt 2):1100-1103

11 Wiersma R. Urethro-ejaculatory duct reflux in children: an updated review. Eur J Pediatr Surg 2009;19(6):374-376

12 Vincent MV, Mackinnon E. The response of clinical balanitis xerotica obliterans to the application of topical steroid-based creams. J Pediatr Surg 2005;40(4):709-712

13 Wilkinson DJ, Lansdale N, Everitt LH, et al. Foreskin preputioplasty and intralesional triamcinolone: a valid alternative to circumcision for balanitis xerotica obliterans. J Pediatr Surg 2012; 47(4):756-759 